Eur. J. Clin. Chem. Clin. Biochem.

Vol. 32, 1994, pp. 859-863

(C) 1994 Walter de Gruyter \& Co. Berlin - New York

\title{
Methodology-Dependent Variations in Reticulocyte Counts Using a Manual and Two Different Flow Cytometric Procedures
}

\author{
By A. J. van Houte', P. C. M. Bartels', M. Schoorl ${ }^{1}$ and C. Mulder ${ }^{2}$
}

1 Department of Clinical Chemistry, Haematology and Immunology, Medical Centre Alkmaar; Alkmaar, The Netherlands,

2 Department of Clinical Chemistry, Free University Hospital, Amsterdam, The Netherlands

(Received April 11/August 15, 1994)

Summary: This paper describes a comparison between the microscopic brilliant cresyl blue and the flow cytometric thiazol orange (FACScan) and auramine $O$ (Sysmex) methods for enumeration of reticulocytes.

The mean intra-assay coefficients of variation for the microscopic, FACScan and Sysmex methods were established to be 33, 9 and 5\% respectively. A rather poor correlation was observed between the microscopic count and both flow cytometric methods (FACScan $r=0.61$; Sysmex $r=0.57$ ). However, the correlation between the flow cytometric methods was satisfactory $(r=0.79)$.

Reference ranges for the reticulocyte count, corresponding with the 2.5th and 97.5th percentile, were determined for the microscopic (8-30\%o), FACScan (11-27\%) and Sysmex (8-18\%o) methods. Sysmex R-3000 methodology definitely revealed the lowest and narrowest reference range.

In conclusion, because of higher reproducibility, flow cytometric analysis of reticulocytes is an attractive alternative procedure for the microscopic enumeration method.

\section{Introduction}

Reticulocytes are red blood cells with varying amounts of residual RNA. According to the amount of residual RNA, reticulocytes can be discriminated from mature red blood cells. The number of reticulocytes may provide additional information with respect to activity of erytbropoiesis. The reticulocyte count is an important indicator in clinical investigations of subjects with anaemia of various causes, e. g. in bone marrow aplasia and in establishing the effect of therapy, e.g. supplementation with iron or cobalamines (1).

Administration of erythropoietin may stimulate erythropoiesis in cases of anaemia associated with chronic renal failure, rheumatoid arthritis, cancer, prematurity and bone marrow transplantation (2). Therefore, recombinant human erythropoietin can be applied in order to reduce blood transfusion requirements (3). Treatment with erythropoietin has also been shown to be effective for the correction of anaemia in zidovudine-treated subjects infected with human immunodeficiency virus (4). In all these cases, the reticulocyte count is considered to be a useful tool to monitor the effectiveness of erythropoiesis stimulation. Improved sensitivity and precision of automated flow cytometric reticulocyte analysis, in comparison with manual procedures, permits it to be utilized in order to detect minor changes in erythropoiesis due to supplementation of erythropoietin $(5,6)$.

The microscopic method for enumeration of reticulocytes using a supravital stain such as brilliant cresyl blue is rather time consuming. Other drawbacks from the manual procedure are the variable distribution of reticulocytes in the blood smear, the small number of reticulocytes which has been actually counted and the large inter-individual variation between technologists in mor- 
phologic identification and enumeration of reticulocytes (7).

The availability of flowcytometers in routine hospital laboratories has provided an attractive tool for automated determination of the reticulocyte count $(8-14)$. By using flow cytometry one has the obvious advantage of being able to classify a larger number of cells in a shorter period of time as compared to manual methods, resulting in a rather low statistical inter-operator variation. Thiazole orange and auramine $\mathrm{O}$ are most frequently used as dyes for pretreatment of red blood cells before flow cytometric reticulocyte enumeration. These membrane-permeable fluorochromes tightly bind to nucleic acids by forming a fluorescent nucleotide-reagent complex, which can be analyzed by flow cytometry (15).

Results of flow cytometric methods using thiazole orange (FACScan) and auramine $\mathrm{O}$ (Sysmex) were compared with each other and with the microscopic brilliant cresyl blue method. Reference values were separately established for each method.

\section{Materials and Methods}

Blood samples were supplied with tripotassium EDTA as an anticoagulant. Samples were collected from subjects with requests for reticulocyte counts and from 100 apparently healthy blood-donors ( 52 males and 48 females) who had not donated blood for at least 9 months. The latter category of subjects was selected in order to establish reference values.

\section{Microscopic method}

Supravital staining of unfixed blood cells was performed by mixing $100 \mu \mathrm{l}$ whole blood with $100 \mu \mathrm{l}$ brilliant cresyl blue (concentration $10 \mathrm{~g} / \mathrm{l}$; Sarstedt B. V., Etten-Leur, The Netherlands), followed by incubation at room temperature for 30 minutes. Subsequently two wedge smears were made and reticulocytes were counted separately by two technologists in various regions amounting to 500 red cells.

\section{FACScan}

Commercially available ready-to-use thiazole orange reagent (Retic-COUNT $0.1 \mathrm{mg} / \mathrm{l}$ thiazole orange in phosphate-buffered saline. Becton Dickinson B. V., Etten-Leur, The Netherlands) was used. Staining of reticulocytes was performed by adding $5 \mu \mathrm{l}$ whole blood to $1 \mathrm{ml}$ Retic-COUNT. The mixture was incubated in polypropylene tubes in the dark at room temperature for 2 hours. Flow cytometry was performed on a FACScan (Becton Dickinson B. V., Etten-Leur, The Netherlands). Data acquisition and analysis was performed using Retic-COUNT software version 2.2 (Becton Dickinson). An analysis gate was automatically set around the red blood cell population on a forward-scatter versus side-scatter dot plot. The fluorescence threshold was set automatically to separate autofluorescence of mature red cells from the fluorescent signal of positively stained reticulocytes.

\section{Sysmex R-3000}

Blood samples are automatically aspirated, diluted and stained with the RNA-binding fluorescent dye auramine $O$. Individual cells are characterized on the basis of the amount of forward light scatter combined with their fluorescence intensity. The instrument was calibrated according to the method recommended by the manufacturer (TOA Medical Electronics Co., Charles Goffin, IJsselstein, The Netherlands).

\section{Statistical analysis}

Comparison of the three reticulocyte enumeration methods was performed by using the orthogonal regression method according to Deming (16).

Reference ranges were estimated by application of non-parametric statistical techniques. For cut-off values, we used the values corresponding with the 2.5 th and 97.5 th percentile.

\section{Results}

\section{Reproducibility}

The intra-assay coefficient of variation for the microscopic and both flow cytometric methods was assessed by calculations on results obtained from duplicate analyses of blood samples with reduced, normal and slightly increased reticulocyte counts. Coefficients of variation on these three levels were established to be 41,32 and $25 \%$ respectively using the microscopic method. For the FACScan methodology, coefficients of variation amounted to 10,9 and $7 \%$ for the indicated reticulocyte levels. Concerning duplicate results of the Sysmex R3000 , coefficients of variation were estimated to 7,5 and $3 \%$ for samples with decreased, normal and sightly increased reticulocyte counts, respectively. The mean intra-assay coefficients of variation for the microscopic, FACScan and Sysmex R-3000 method were established to be 33,9 and $5 \%$ respectively.

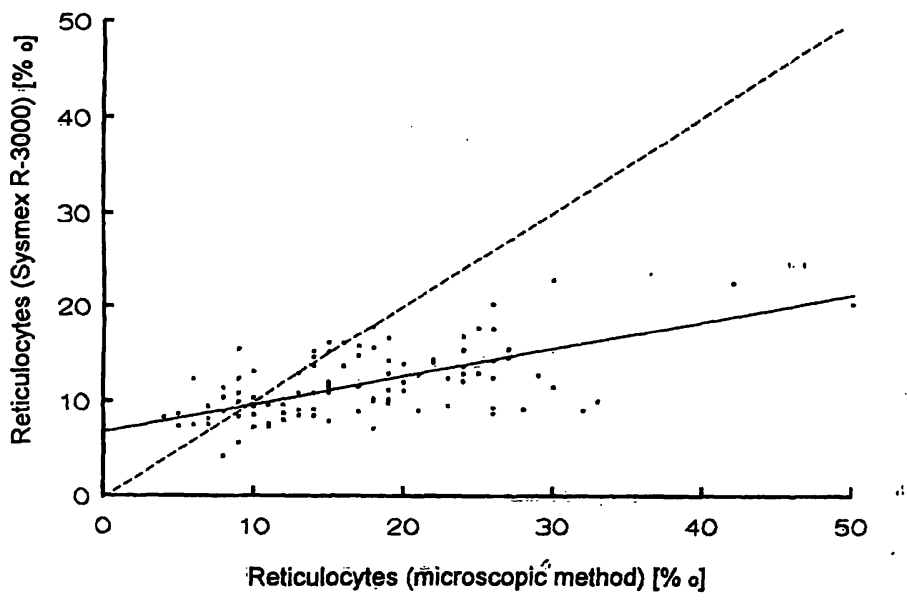

Fig. 1 Relationship, expressed by orthogonal regression line $(y=0.28 x+6.9)$ and coefficient of correlation $(r=0.57)$, between the microscopic and Sysmex R-3000 methods for reticulocyte enumeration in 100 healthy ajfults ( 52 men and 48 women). 


\section{Deviations in reticulocyte counts due to test methodology}

Reticulocyte counts obtained by the three methods were compared in 100 samples of healthy adults. The relationship between the various methods together with the orthogonal regression line and coefficient of correlation ( $r$ ) are shown in figure 1,2 and 3. Rather poor coefficients of correlation were observed between the microscopic count and the FACScan $(r=0.61)$ or the Sysmex $R$ $3000(r=0.57)$. The correlation between the flow cytometric methods was satisfactory $(r=0.79)$.

\section{Frequency distribution histograms and reference ranges}

The reticulocyte count was determined by microscopy and both flow cytometric methods in blood samples obtained from 100 healthy adults ( 52 men and 48 women).

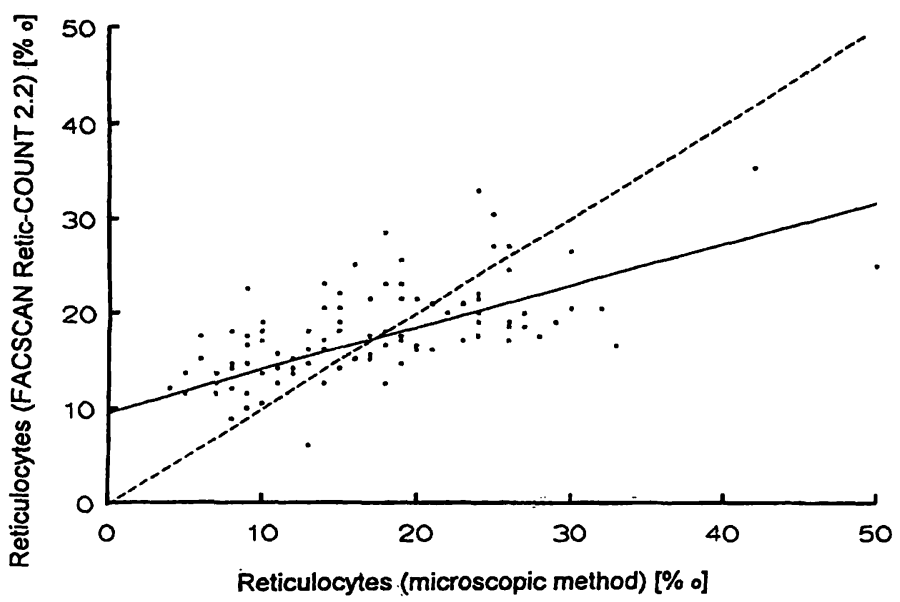

Fig. 2 Relationship, expressed by orthogonal regression line $(y=0.47 x+9.8)$ and coefficient of correlation $(r=0.61)$, between the microscopic and FACScan methods for reticulocyte enumeration in 100 healthy adults ( 52 men and 48 women).

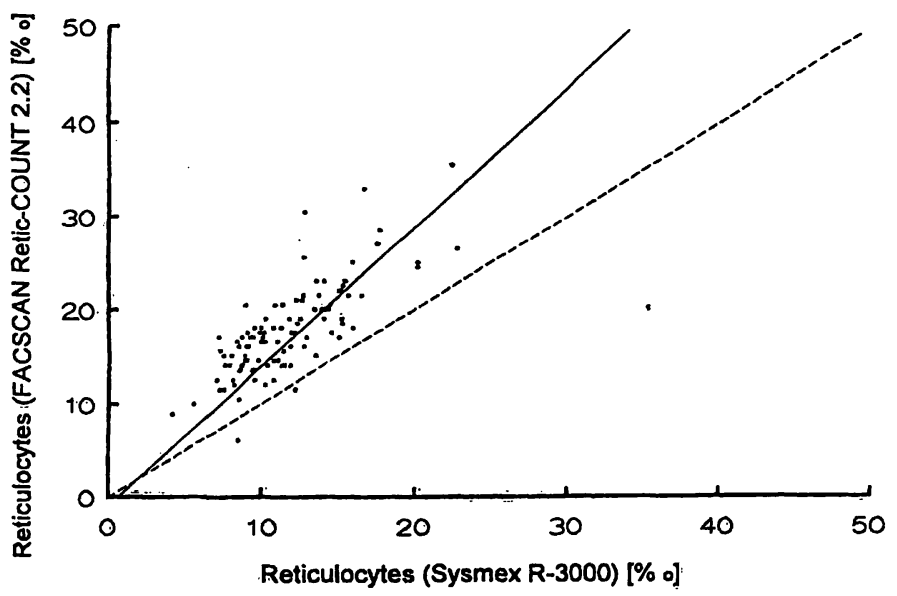

Fig. 3 Relationship, expressed by orthogonal regression line $(y=1.57 x-0.5)$ and coefficient of correlation $(r=0.79)$, between the Sysmex R-3000 and FACScan methods for reticulocyte enumeration in 100 healthy adults ( 52 men and 48 women).

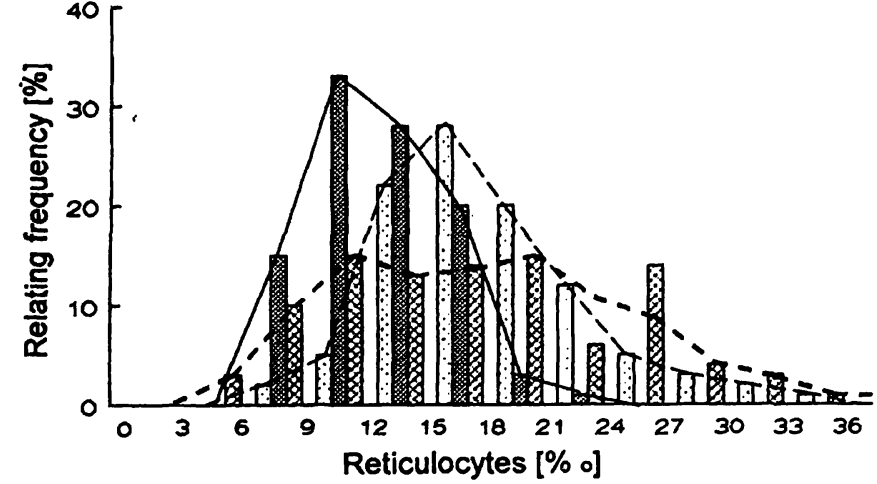

Fig. 4 Frequency distribution histograms for reticulocyte counts in 100 healthy adults ( 52 men and 48 women) obtained by FACScan, Sysmex R-3000 and microscopic methods.

FACScan Sysmex Microscopic method

Frequency distribution histograms concerning different methodologies for reticulocyte enumeration are depicted in figure 4. From the histograms it is obvious that the distribution of the results of apparently healthy subjects is not Gaussian, because a skewness towards higher results is observed. Sysmex R-3000 reticulocyte counts show a narrower frequency distribution histogram with an obvious shift towards lower values compared to both the FACScan and microscopic method. Reference ranges for reticulocytes, corresponding with the 2.5th and 97.5th percentile, are shown in table 1. Sysmex R-3000 methodology definitely revealed the lowest and narrowest reference range.

\section{Discussion}

Microscopic reticulocyte counting is one of the classical methods in haematology (7). Drawbacks of the microscopic method are the rather subjective assessment of the number according to the stage of maturation of the red blood cells and the elaborate and imprecise counting procedure. In particular, microscopic counting does not permit accurate enumeration of abnormally low reticulocyte levels owing to the large analytical variability.

Application of flow cytometric reticulocyte enumeration methods has obviously resulted in improved reproducibility. However, various automated and semi-automated

Tab. 1 Reference ranges for the reticulocyte count, corresponding with the 2.5 th and 97.5 th percentile, obtained by different enumeration methods in 100 healthy adults ( 52 men and 48 women).

\begin{tabular}{lc}
\hline Method & $\begin{array}{l}\text { Reticulocyte } \\
\text { reference range (\%o) }\end{array}$ \\
\hline Microscopy & $8-30$ \\
FACScan & $11-27$ \\
Sysmex R-3000 & $8-18$ \\
\hline
\end{tabular}


counting techniques which are currently used for reticulocyte enumeration, yield deviating results $(1,9,11$, $14,17)$.

A major problem for the analysis of reticulocytes based on the RNA content is caused by the continuous loss of RNA during maturation of the reticulocyte to a red blood cell. Objective criteria for discriminating reticulocytes other than characteristics concerning reticulocyte morphology are not available. That is a reason why striking discrepancies between different methodologies applied for reticulocyte analysis may exist. On the other hand, deviations between results obtained with different methods may be due to interference from other cells. For instance, in flow cytometric techniques using thiazole orange for reticulocyte analysis a light scatter gate around the red blood cell population is utilized to minimize platelet and leukocyte contamination of the collected fluorescence signal (5). Drawbacks of flow cytometric reticulocyte analysis using thiazole orange are the reported errors due to contamination with Howell-Jolly bodies or nucleated red blood cells and variances in staining conditions $(9,14,18)$. However, in this study the higher reticulocyte counts obtained by FACScan compared to Sysmex R-3000 (tab. 1, fig. 3) are very likely not due to inclusions in red blood cells because our population consists of healthy adults ( 52 men and 48 women).

Most reports concerning evaluation of automated reticulocyte counting procedures yield data comparable to the manual microscopic method $(10,11,15)$. However, in our investigations only poor coefficients of correlation could be established between the microscopic counting technique and both flow cytometric procedures in apparently healthy subjects (fig. 1 and 2). Probably, the rather

\section{References}

1. Hansson, G. K., Andersson, M., Jarl, H. \& Stemme, S. (1992) Flow cytometric analysis of reticulocytes using an RNA-binding fluorochrome. Scand. J. Lab. Invest. 52, 35-41.

2. Kanold, J., Bezou, M. J., Coulet, M., Quainon, F., Malpuech, G., Travade, Ph. \& Deméocq, F. (1993) Evaluation of erythropoietic/hematopoietic reconstitution after BMT by highly fluorescent reticulocyte counts compares favorably with traditional peripheral blood counting. Bone Marrow Transpl. 11, 313318.

3. Ayash, L. J., Elias, A., Hunt, M., Demetri, G., Wheeler, C., Tepler, J., Schwartz, G., Mazanet, R., Reich, E., McCauley, M., Antman, K. \& Anderson, K. C. (1994) Recombinant human erythropoietin for the treatment of anemia associated with autologous bone marrow transplantation. Brit. J. Haemat. 87 , 153-161.

4. Phair, J. P., Abels, R. I., McNeill, M. V. \& Sullivan, D. J. (1993) Recombinant human erythropoietin treatment: Investigational new drug protocol for the anemia of the acquired immunodeficiency syndrome. Arch. Intern. Med. 153, 26692675. poor correlation is due to the large mean coefficient of variation $(33 \%)$ of the microscopic counts. Proficiency testing programs regarding manual reticulocyte counting methodologies have documented coefficients of variation between different institutes in the range of 40 to $60 \%$ (5). In contrast, a considerably better coefficient of correlation was calculated after comparison of the Sysmex R-3000 with the FACScan (fig. 3).

The Sysmex R-3000 methodology revealed the narrowest frequency distribution histogram of reticulocyte counts (fig. 4). As a consequence, for the Sysmex R3000 the reference range established for apparently healthy subjects is rather narrow $(8-18 \%$, tab. 1) and in agreement with earlier observations $(17,19)$. Several factors should be further investigated in order to elucidate causes for discrepancies between counting procedures.

In conclusion, flow cytometric counting of reticulocytes is an attractive alternative procedure to the microscopic enumeration method. Statistically, the analytical precision of especially the Sysmex R-3000 is significantly better than for the microscopic method. Therefore Sysmex R-3000 allows a more reliable estimation of abnormally low reticulocyte levels. This flow cytometric methodology can be very well utilized to detect minor changes in erythropoiesis due to suppletion of erythropoietin. An additional advantage of the automated flow cytometric methods is the reduction of laboratory technician time.

\section{Acknowledgement}

Miss $K$. Lagerwaard is gratefully acknowledged for excellent secretarial assistance.
5. Davis, B. H. \& Bigelow, N. C. (1993) Flow cytometric reticulocyte analysis and the reticulocyte maturity index. Ann. N. Y. Acad. Sci. 677, 281-292.

6. Brugnara, C., Colella, G. M., Cremins, J., Langley, R. C., Schneider, T. J., Rutherford, C. J. \& Goldberg, M. A. (1994) Effects of subcutaneous recombinant human erythropoietin in normal subjects: Development of decreased reticulocyte hemoglobin content and iron-deficient erythropoiesis. J. Lab. Clin. Med. $123,660=667$.

7. Koepke, J. F. \& Koepke, J. A. (1986) Reticulocytes. Clin. Lab. Haematol. 8, 169-179.

8. Tanke, H. J. (1992) Reticulocytes and mature erythrocytes. In: Flow cytometry in hematology (Laerum, O. D. \& Bjerkness, R., eds.) Academic Press, London, pp. 75-93.

9. Van Hove, L., Goossens, W., van Duppen, V. \& Verwilghen, R. L. (1990) Reticulocyte count using thiazole orange. A flow cytometry method. Clin. Lab. Haematol. 12, 287-299.

10. Nobes, P. R. \& Carter, A. B. (1990) Reticulocyte counting using flow cytometry. J. Clin. Pathol. 43, 675-678. 
11. Ferguson, D. J., Lee, S. F. \& Gordon, P. A. (1990) Evaluation of reticulocyte counts by flow cytometry in a routine laboratory. Am. J. Haematol. 33, 13-17.

12. Tichelli, A., Gratwohl, A., Driessen, A., Matthys, S., Pfefferkorn, E., Regenass, A., Schumacher, P., Stebler, C., Wernli, M., Nissen, C. \& Speck, B. (1990) Evaluation of the Sysmex $\mathrm{R}-1000$. An automated reticulocyte analyser. Am. J. Clin. Pathol. 93, 70-78.

13. Schimenti, K. J., Lacerna, K., Wamble, A., Maston, L., Iaffaldano, C., Straight, M., Rabinovitch, A., Lazarus, H. M. \& Jacobberger, J. W. (1992) Reticulocyte quantification by flow cytometry, image analysis and manual counting. Cytometry 13, 853-862.

14. Lofsness, K. G., Kohnke, M. L. \& Geier, N. A. (1994) Evaluation of automated reticulocyte counts and their reliability in the presence of Howell-Jolly bodies. Am. J. Clin. Pathol. $101,85-90$.

15. Lee, L. G., Chen, C. H. \& Chin, L. A. (1986) Thiazole orange: A new dye for reticulocyte analysis. Cytometry 7, 508-517.

16. Cornbleet, P. J. \& Gochman, N. (1979) Incorrect lcast-squares regression coefficients in method-comparison analysis. Clin. Chem. 25, 432-438.
17. Hohenwallner, W., Wiesinger, K. \& Wimmer, E. (1992) The reticulocytes: Automatic counting, indication and interpretation. Sysmex J. Int. 2, 120-135.

18. Davis, B. H., DiCorato, M., Bigelow, N. C. \& Langweiler, M. H. (1993) Proposal for standardization of flow cytometric reticulocyte maturity index (RMI) measurements. Cytometry $14,318-326$

19. Bowen, D., Bently, N., Hoy, T. \& Cavill, I. (1991) Comparison of a modified thiazole orange technique with a fully automated analyser for reticulocyte counting. J. Clin. Pathol. 44, 130133.

Dr. A. J. van Houte

Department of Clinical Chemistry,

Haematology and Immunology

Medical Centre Alkmaar

P.O. Box 501

NL-1800 AM Alkmaar

The Netherlands 
